САХАРНЫЙ ДИАБЕТ И БЕРЕМЕННОСТЬ. ЧАСТЬ ІІ. ТЕЧЕНИЕ, ТАКТИКА ВЕДЕНИЯ ПАЦИЕНТОВ

\author{
'Никонова Л.B. (lola.nikonova.58@mail.ru),'Тишковский C.B. (tishkovsky@rambler.ru), \\ 2Гадомская B.И. (ver.gadomskaia@gmail.com),'Давыдчик Э.B. (davydchike@mail.ru), \\ Гулинская О.B. (gulinskaya@gmail.com) \\ 'УО «Гродненский государственный медицинский университет», Гродно, Беларусь \\ 2 ГУЗ «Городская поликлиника N4», Гродно, Беларусь
}

Введение. Сахарный диабет (СД) занимает одно из первых мест в мире по распространенности и наличию осложнений. Одним из факторов, влияющих на течение сахарного диабета и его осложнений, является беременность.

Цель: изучение особенностей течения СД и его осложнений при беременности и тактика ведения беременных с СД в зависимости от срока гестации, течения СД и наличия его осложнений.

Материал и методы: проанализированы 34 литературных источника по данной тематике отечественных и зарубежных авторов.

Результаты. Установлено, что течение СД при беременности носит лабильный характер и способствует прогрессированию сосудистых осложнений у матери. Строгий контроль гликемии, введение режима многоразовых инъекций инсулина оказывает благоприятное воздействие на течение беременности и рождение здорового ребенка.

Bblводы: применение интенсифицированной инсулинотерапии при ведении беременных с СД, усовершенствование тактики ведения и лечения беременных, использование современных технологий дало возможность улучшить исходы беременности для матери и плода: снизилась частота тяжельх форм гестоза, многоводия, преждевременных родов, перинатальную смертность.

Ключевые слова: сахарный диабет, беременность, гипергликемия, гипогликемия, инсулинотерапия, лечение.

Течение сахарного диабета (СД) при беременности отличается волнообразным характером и повышенной склонностью к кетоацидозу и гипогликемии. Такое лабильное течение СД способствует прогрессированию сосудистых осложнений. Рассмотрим некоторые из них.

Диабетическая нефропатия (ДН) - это прогрессирующее осложнение, которое поражает от 30 до 40\% пациентов с СД. Существуют четыре фактора, связанные с беременностью, которые могут увеличить риск развития нефропатии. Во-первых, во время нормальной беременности уровень клубочковой фильтрации увеличивается на 40-60\%. Во-вторых, артериальная гипертензия (АГ) и преэклампсия, возникающие на фоне беременности, встречаются у $15-20 \%$ женщин с СД и могут оказать негативное влияние на функцию почек. В-третьих, диета с большим содержанием белка может привести к увеличению клубочковой фильтрации. Таким образом, большее содержание белка в пище, которое необходимо беременным, может потенцировать клубочковую гиперфильтрацию и ускорить естественное течение диабетической нефропатии. И в-четвертых, так как ингибиторы АПФ, замедляющие прогрессирование нефропатии, неблагоприятно влияют на беременность, эти препараты отменяют во время беременности [3, 13].

Строгий контроль гликемии, рекомендуемый во время беременности, может оказать благоприятное воздействие на течение нефропатии [3, $5,6]$. ДН в свою очередь тоже оказывает влияние на исход беременности. У $50 \%$ женщин с ДН развивается преэклампсия. У женщин с нефро- патией увеличен риск преждевременных родов из-за ухудшения состояния женщины или угрозы ребенку. Примерно в 25-30\% таких беременностей роды наступают до 34-й недели, а в $50 \%$ случаев роды происходят до 37-й недели гестации. В 20\% беременностей на фоне нефропатии наблюдается истощение или недоразвитие плода $[3,4,13,17,33]$.

Диабетическая ретинопатия - это микрососудистое осложнение СД с поражением сосудов сетчатки (развитием микроаневризм, кровоизлияний), проявляющееся экссудативными изменениями сетчатки, макулопатией и пролиферативным ростом новообразованных сосудов. Прогрессирование ретинопатии зависит от исходной стадии заболевания. При отсутствии ретинопатии или при непролиферативной стадии ухудшение со стороны сосудов сетчатки глаза происходит реже, чем при других стадиях ретинопатии. Качество контроля гликемии во время зачатия и беременности, а также резкие колебания гликемии с гипогликемическими эпизодами прямо связаны с прогрессированием ретинопатии [5]. Предполагается, что постепенное достижение адекватного контроля гликемии при планировании беременности предотвратит развитие ретинопатии. Установлено, что у 50-60\% женщин с исходной $А Г$ или с $А Г$, возникшей во время беременности, происходит прогрессирование ретинопатии. В отличие от нефропатии наличие ретинопатии само по себе не оказывает неблагоприятных эффектов на исход беременности. Некоторые женщины имеют сочетание ретинопатии и нефропатии, но в этой группе пациенток повышенный риск неблагоприятного исхода 
беременности в большей степени связан с нефропатией, а не с ретинопатией $[1,3,4,13,18]$. В послеродовом периоде происходит регресс изменений сетчатки $[3,4]$.

Диабетическая нейропатия - патогенетически связанное с СД сочетание синдромов поражения нервной системы, классифицируемое в зависимости от преимущественного вовлечения в процесс спинномозговых нервов (дистальная и периферическая нейропатия) и вегетативной нервной системы (висцеральная, или автономная, диабетическая нейропатия). Недостаточно данных о взаимном влиянии диабетической нейропатии и беременности. При долговременном наблюдении установлено, что беременность не способствует возникновению или ухудшению течения данного осложнения [3]. Особое значение при беременности имеет наличие диабетической автономной нейропатии с парезом желудка, так как происходит усиление тошноты и рвоты. Это может привести к нарушению абсорбции питательных веществ, неадекватному питанию и нарушению контроля гликемии. На естественное течение диабетической автономной нейропатии беременность не влияет $[3,31]$.

Следующим осложнением СД при беременности является гипогликемия. Гипогликемия возникает преимущественно во время первого триместра беременности, но не считается в настоящее время тератогенным фактором, то есть она не способствует развитию пороков и уродств у плода $[3,11,20]$.

На фоне СД у беременной женщины увеличивается риск спонтанного аборта. Это связано с токсическим воздействием гипергликемии и кетоацидоза на развивающийся эмбрион, что может привести к дегенерации эмбриона и появлению пустого плодного яйца (анэмбрионии) или пороков развития, несовместимых с пролонгированием беременности. Другие возможные причины спонтанного аборта при плохом контроле гликемии - аномальная плацентация и васкуляризация, более высокая частота хромосомных аномалий $[3,11]$. До сих пор обсуждается вопрос о существовании порога гликемии, после которого повышается риск спонтанного аборта у женщин с СД. Однако в исследовании «Диабет на ранних этапах беременности» (DIEP) пороговый эффект гликемии не обнаружен, но установлено, что чем больше был уровень $\mathrm{HbA1c} \mathrm{в}$ I триместре беременности, тем больше риск возникновения спонтанного аборта. Таким образом, очевидно, что улучшение контроля гликемии до зачатия уменьшает риск спонтанного аборта [3, 28].

В нескольких исследованиях установлена взаимосвязь между появлением врожденных пороков развития (ВПР) и плохого контроля гликемии у женщин с СД (HbA1c $>8 \%$ перед зачатием и в течение I триместра беременности повышает риск развития ВПР в 2-4 раза по сравнению с частотой в общей популяцией беременных женщин) [24]. Большинство пороков развития формируется до 7-й недели беременности. Инсулин не проходит через плаценту, а плод до 8-12 не- дели беременности не вырабатывает собственный инсулин. Поступление от матери к плоду большого количества глюкозы в этот период приводит к выраженному нарушению обменных процессов, имеющих тератогенный эффект. Наиболее часто отмечаются пороки сердца, аномалии ЦНС, гипо- и агенезия каудального отдела позвоночника, поликистоз почек. Некоторые авторы считают, что риск возникновения ВПР увеличивается при наличии диабетической ангиопатии у матери $[3,8,20,21]$.

Неблагоприятное влияние СД матери на плод проявляется формированием у него симптомокомплекса - диабетической фетопатии, которая отмечается характерным внешним видом, быстрым увеличением массы тела, высокой частотой пороков развития, функциональной незрелостью органов и систем плода, отклонением от нормального течения периода новорожденности, высокой перинатальной смертностью. У беременных с СД 1 типа и сосудистыми осложнениями частота диабетической фетопатии достигает 75,5\% [8].

По своему внешнему виду новорожденные напоминают пациентов с синдромом Иценко-Кушинга: цианоз, отечность, большой живот и чрезмерно развитый подкожный жировой слой, лунообразное лицо, большое количество петехий и кровоизлияний на коже лица и конечностей, выраженный гипертрихоз. Характерна непропорциональность телосложения: длинное туловище, короткая шея, маленькая голова. Окружность головы значительно меньше окружности плечевого пояса [8].

Макросомия - основной признак диабетической фетопатии, имеется у $8-43 \%$ плодов и объясняется чрезмерным накоплением жировой ткани, составляющей более $20 \%$ массы тела (у плодов здоровых матерей - до 16\%). Такие младенцы имеют высокий риск родовых травм, послеродовой гипогликемии (50\%), так как сразу после рождения прекращается трансплацентарный транспорт глюкозы к плоду, а концентрация инсулина в крови остаётся высокой, а также риск гипербилирубинемии (6-50\%), полицитемии.

У потомства матерей с сосудистыми осложнениями диабета и поздним гестозом макросомия практически не встречается. В этих случаях более характерно отставание массы плода от возрастной нормы, хотя другие проявления фетопатии сохраняются [4, 10, 16, 23, 27].

Вследствие повышенной утилизации глюкозы плодом в ранние сроки беременности наблюдается снижение потребности в инсулине у матери. Присоединение раннего токсикоза и связанное с ним уменьшение потребления пищи увеличивает вероятность гипогликемии [9, 25]. При беременности меняются ощущения гипогликемии, учащаются бессимптомные гипогликемии, особенно в ночное время. Возникает необходимость более частого измерения уровня глюкозы в крови с целью адекватного и своевременного изменения доз инсулина. Поэтому уровень глюкозы в крови нужно определять несколько раз в сутки. При коррекции доз инсули- 
на ориентируются на уровни глюкозы в крови натощак. Кроме того, необходимо следить за уровнем глюкозы в крови после приема пищи. Это позволяет предупредить макросомию плода [8].

Во второй половине беременности потребность в инсулине постепенно возрастает, достигая максимальных значений в 33 недели (140-160\%). С 35-й недели беременности наблюдается постепенное снижение потребности в инсулине. Снижение потребности в инсулине может начаться раньше при выраженной плацентарной недостаточности, которая часто встречается у пациентов с сосудистыми осложнениями СД и может потребовать досрочного прерывания беременности. Потребность в инсулине остается низкой в течение первых двух дней послеродового периода. Начиная с третьего дня после родов, потребность в инсулине начинает возрастать и через 3-4 дня достигает исходного уровня [2, 9].

\section{Тактика ведения беременности при СД}

\section{Диетотерапия}

Вопросы соблюдения диеты у беременных с СД остаются предметом споров. После многих лет использования низкокалорийной диеты в 1979 г. American Diabetic Association (ADA) предложила нормокалорийную диету, состоящую из 20\% белков, менее $30 \%$ жиров и 50-60\% углеводов с ограничением холестерина, насыщенных жирных кислот и богатую волокнами [12]. В последних рекомендациях содержание белков остается неизменным, а оставшаяся часть распределяется между жирами и углеводами так, что менее $10 \%$ составляют насыщенные жирные кислоты и не более $10 \%$ - полиненасыщенные жирные кислоты, 60-70\% калорий приходится на мононасыщенные жирные кислоты и углеводы. Диета предлагает 6 приемов пищи в день: 3 основных и 3 дополнительных - утром, в полдень и перед сном (для предотвращения ночной гипогликемии) [14]. В основном считается, что беременность - не самое подходящее время для уменьшения массы тела. Не рекомендуется очень строгая диета, так как она может стать причиной возникновения кетоацидоза, который ассоциирован со снижением интеллекта у будущего потомства. Peterson и Jovanovic предлагают следующую диету для беременных с СД: 30 ккал на 1 кг фактической массы тела для беременных женщин с нормальной массой тела; 35-40 ккал/ кг для женщин с дефицитом массы тела; 24 ккал/ кг для женщин с избыточной массой тела и 12 ккал/кг для женщин с ожирением [14, 25].

Инсулинотерапия

Во время беременности инсулины применяются как при СД 1 типа, так и при СД 2 типа. В течение беременности потребность в инсулине быстро меняется. Схемы инсулинотерапии и дозы также меняются в зависимости от типа СД и индивидуальных потребностей пациентки. При СД 1 типа используется интенсифицированная базис-болюсная инсулинотерапия в режиме многократных подкожных инъекций инсулина
(МПИИ), включающая одну и больше инъекций инсулина средней длительности действия и инъекции инсулина короткого действия или инсулина ультракороткого действия перед каждым основным приемом пищи. Средняя суточная потребность инсулина составляет 0,86 ед/кг в I триместре беременности; 0,95 ед/кг во II триместре и 1,2 ед/кг в III [3, 9, 25].

Для дополнительной коррекции гипергликемии частота инъекций инсулина короткого действия, особенно инсулина ультракороткого действия, может увеличиваться при беременности до 5-6 и более раз в сутки. Прежде всего это обусловлено тем, что большинству женщин в данный период требуется дополнительная инъекция инсулина короткого действия в ранние утренние часы для компенсации гипергликемии, обусловленной феноменом «утренней зари». Кроме того, во время беременности особенности фармакокинетики и фармакодинамики инсулина по целому ряду причин препятствуют достижению целевой гликемии после приема пищи (целевые значения гликемии во время беременности: глюкоза плазмы натощак, перед едой, перед сном, 3 часа ночи $<5,1$ ммоль/л; глюкоза плазмы через 1 час после еды $<7,0$ ммоль/л; HbA1c <6,0\%) [6]. Обычно пик действия инсулина короткого действия наступает через 2-3 часа после его введения, тогда как у беременных самая высокая постпрандиальная гликемия регистрируется через 1-1,5 часа после приема пищи. Введение больших доз инсулина короткого действия перед едой может привести к резкому снижению гликемии через 3-4 часа после приема пищи. Во избежание гипогликемии в эти часы требуется дополнительный прием пищи, что в свою очередь провоцирует гипергликемию перед следующим приемом пищи и способствуют нежелательной прибавке в весе [7, 34]. Кроме того, инсулины короткого действия необходимо вводить за 20-30 мин., а во II и III триместрах за 40-60 мин. до приема пищи, что создает дополнительные неудобства для беременной и снижает качество ее жизни. В отличие от инсулина короткого действия, инсулин ультракороткого действия можно вводить непосредственно перед приемом пищи, а при необходимости (ранний токсикоз беременных) и после еды. Максимальное действие инсулина ультракороткого действия приходится именно на пик постпрандиальной гипергликемии при беременности (1-1,5 часа от начала приема пищи), что снижает вариабельность гликемии в течение суток и частоту тяжелых гипогликемий $[7,22]$.

В качестве базиса используют пролонгированный инсулин средней продолжительности действия в виде 1-2 кратных инъекций в течение суток. Аналоги инсулинов пролонгированного действия во время беременности не назначаются, так как пока нет долгосрочных исследований и данных по отсутствию тератогенного эффекта $[4,15,19,30,32]$.

Альтернативой МПИИ является помповая инсулинотерапия или постоянная подкожная инфузия инсулина (ППИИ). Все преимущества 
этого способа введения инсулина оказались особо важными при беременности. При режиме ППИИ доставка инсулина в организм осуществляется инсулиновым дозатором (помпой) по базис-болюсному принципу. При этом используется только один тип инсулина - инсулин ультракороткого действия, непрерывное поступление которого в кровь обеспечивает постоянно меняющуюся потребность пациента в инсулине.

Базисный принцип работы помпы основывается на круглосуточной автоматической подаче микродоз инсулина ультракороткого действия каждые несколько минут (в зависимости от используемой модели инсулиновой помпы), что максимально точно имитирует работу здоровой поджелудочной железы. С учетом индивидуальных потребностей в инсулине в течение суток инсулиновая помпа позволяет программировать сразу несколько базальных профилей. Это снижает риск развития гипогликемии в ночное время и вне приема пищи, а также позволяет избежать «феномена утренней зари». Метод разрешает пациенту в любой момент времени самостоятельно увеличить или уменьшить количество поступающего инсулина, например, в период болезни или при повышенной физической активности. Идеальное распределение общей дозы инсулина в течение суток и отсутствие его подкожного депо (как при МПИИ) уменьшают суточную потребность в инсулине [7, 26, 29].

При использовании помпы постпрандиальные колебания гликемии нивелируются болюсным введением инсулина, которое программируется индивидуально в зависимости от вида принимаемой пищи.

«Нормальный» болюс обычно вводится на тот прием пищи, в котором явно преобладают углеводы, а также с целью снижения выявленной при самоконтроле гипергликемии.

«Пролонгированный» болюс позволяет вводить запрограммированную дозу в период выбранного времени от 30 минут до восьми часов.

«Многоволновой», или болюс «двойной волны», включает оба предыдущих болюса («нормальный» и «пролонгированный»), долевое распределение которых индивидуально рассчитывается пациентом в зависимости от состава планируемой пищи.

«Пролонгированный» и «многоволновой» болюсы незаменимы при сложном составе пищи, богатой белками и жирами, гастропарезе, раннем токсикозе беременных, а также при необходимости постепенного снижения гипергликемии [26].

Несомненным преимуществом является уменьшение локально-зависимой вариабельно-

\section{Литература}

1. Арбатская, Н. Ю. Сахарный диабет и беременность / Н. Ю. Арбатская // Фарматека. - 2000. - № 5. C. 30-36.

2. Аржанова, О. Н. Особенности течения беременности и родов при сахарном диабете в современных условиях / О. Н. Аржанова, Н. Г. Кошелева // Журнал акушерства и женских болезней. - 2006. - T. LV, № 1. - С. 12-16. сти всасывания инсулина из места инъекции, так как инфузия инсулина в течение двух-трех дней производится в одно и то же место.

Переводить на помповую инсулинотерапию можно только обученных, хорошо ориентирующихся в лечении СД и, что особо важно, высоко мотивированных именно на данный вид терапии пациенток [7].

Одним из немногих побочных явлений, связанных с применением ППИИ, является риск быстрого развития кетоацидоза на фоне технической неисправности инсулинового дозатора (неадекватная работа помпы, окклюзия или отсоединение катетера) или инфицирования кожных покровов [7].

Госпитализировать беременных с СД в эндокринологическое отделение рекомендуется:

1. На раннем сроке беременности для обследования, решения вопроса о сохранении беременности, более точного определения срока беременности, проведения профилактических мероприятий, компенсации диабета, обучения в «школе диабета».

2. При сроке беременности 21-24 недели в связи с высокой вероятностью ухудшения течения диабета и появлением осложнений беременности.

3. При сроке беременности 35-36 недель - для тщательного наблюдения за плодом, лечения акушерских и диабетических осложнений, выбора срока и метода родоразрешения [8].

Во время беременности противопоказан прием следующих лекарственных средств:

- таблетированные сахароснижающие препараты;

- ингибиторы АПФ и блокаторы рецепторов ангиотензина II;

- ганглиоблокаторы;

- антибиотики (аминогликозиды, тетрациклины, макролиды и др.);

- статины [6].

\section{Выводы}

Применение интенсифицированной инсулинотерапии при ведении беременных с СД, усовершенствование тактики ведения и лечения беременных, использование современных технологий дало возможность улучшить исходы беременности для матери и плода: снизилась частота тяжелых форм гестоза, многоводия, преждевременных родов, перинатальная смертность. Однако по-прежнему ведение беременных с СД остается сложной задачей, требующей совместных усилий врачей разных специальностей.

3. Планирование беременности у женщин с сахарным диабетом / О. Р. Григорян [и др.] // Вестник репродуктивного здоровья. - 2011. - № 1. - С. 23-31.

4. Данилова, Л. И. Сахарный диабет и беременность : учеб.-метод. пособие / Л. И. Данилова, И. И. Бурко, 3. В. Забаровская. - Минск, [б. и.], 2010. - 34 с.

5. Дедов, И. И. Сахарный диабет: ретинопатия, нефропатия / И. И. Дедов, М. В. Шестакова, Т. М. Миленькая. - Москва : Медицина, 2001. - 176 с. 
6. Алгоритмы специализированной медицинской помощи больным сахарным диабетом : клинические рекомендации / И. И. Дедов [и др.] // Сахарный диабет. 2015. - Т. 18, № 1S. - С. 72-74.

7. Демидова, И. Ю. Актуальные проблемы компенсации сахарного диабета при беременности / И. Ю. Демидова, Н. Ю. Арбатская, Е. П. Мельникова // Сахарный диабет. - 2009. - № 4. - С. 32-38.

8. Захарова, Т. Г. Сахарный диабет и беременность : учеб. пособие для студентов старших курсов лечеб. фак-та / Т. Г. Захарова, М. М. Петрова, Г. П. Лака. Красноярск : Издательские линии, 2006. - 126 с.

9. Потин, В. В. Инсулинотерапия больных сахарным диабетом 1 типа во время беременности / В. В. Потин, Н. В. Боровик, А. В. Тиселько // Сахарный диабет. 2009. - № 1. - С. 39-41.

10. Федорова, М. В. Сахарный диабет, беременность и диабетическая фетопатия : справ. пособие / М. В. Федорова, В. И. Краснопольский, В. А. Петрухин. - Москва : Медицина, 2001. - 288 с.

11. Диабет и беременность: обзор западных клинических рекомендаций UpToDate.com / пер. и коммент. Д. Чернова. -2015 . -118 c.

12. Standards of medical care in diabetes-2014 / American Diabetes Association // Diabetes Care. - 2014. - Vol. 37, syppl. 1. - S14-S80. - doi: 10.2337/dc14-S014.

13. Effects of pregnancy on microvascular complications in the Diabetic Control and Complications Trial / DCCT Research Group // Diabet Care. - 2000. - Vol. 23, iss. 8. - P. 1084-1100.

14. Crowe, S. M. Oral glucose tolerance test and the preparatory diet / S. M. Crowe, J. M. Mastrobattista, M. Monga // Am. J. Obstet. Gynecol. - 2000. - Vol. 182, iss. 5. - P. 1052-1054.

15. Perinatal outcomes associated with the use of glargine in pregnancy / G. Di Cianni [et al.] // Diabet Med. - 2008. - Vol. 25, iss.8. - P. 993-996. - doi: 10.1111/j.14645491.2008.02485.x.

16. Macrosomia despite good glycaemic control in Type I diabetic pregnancy; results of a nationwide study in the Netherlands / I. M. Evers [et al.] // Diabetologia. - 2002. - Vol. 45, iss. 11. - P. 1484-1489. - doi: 10.1007/s00125002-0958-7.

17. Expert consensus document on management of cardiovascular diseases during pregnancy / The Task Force on the Management of Cardiovascular Diseases During Pregnancy of the European Society of Cardiology // Eur. Heart J. - 2003. - Vol. 24, iss 11. - P. 761-781.

18. Benefits, risks, costs, and patients satisfaction associated with insulin pump therapy for the pregnancy complicated by type 1 diabetes mellitus / S. G. Gabbe [et al.] // Am. J. Obstet Gynecol. - 2000. - Vol. 182, iss.6. - P. 1283.

19. Gallen, I. W. Survey of glargine use in 115 pregnant women with type 1 diabetes / I. W. Gallen [et al.] // Diabet Med. - 2008. - Vol. 25, iss. 2. - P. 165-169.

20. Prevalence of nocturnal hypoglycemia in first trimester of pregnancy in patients with insulin treated diabetes mellitus / E. Hellmuth [et al.] // Acta Obs. Gyn. Scand. - 2000. - Vol. 79, iss. 11. - P. 958. - doi: 10.1034/j.16000412.2000.079011958.x.

21. Why don't women with diabetes plan their pregnancies? / E. V. Holing [et al.] // Diabetes Care. - 1998. Vol. 21, iss. 6. - P. 889-895. - https://doi.org/10.2337/ diacare.21.6.889.

22. Improved glycemic control with insulin aspart: a multicenter randomized double-blind crossover trial in type 1 diabetic patients. UK Insulin Aspart Study Group / P. Home [et al.] // DiabetCare. - 1998. - Vol. 21, № 11. - P. 1904-1909. - https://doi.org/10.2337/diacare.21.11.1904.

23. Poor glycatedhaemoglobin control and adverse pregnancy outcomes in type 1 and type 2 diabetes mellitus: Systematic review of observational studies / M. E. Inkster [et al.] // BMC Pregnancy and Childbirth. - 2006. - Vol. 6. - P. 30. - http://doi.org/10.1186/1471-2393-6-30.

24. Diabetes and pregnancy: factors associated with seeking preconception care / N. K. Janz [et al.] // Diabetes Care. - 1995. - Vol. 18, iss. 2. - P. 157-165. - https://doi. org/10.2337/diacare.18.2.157.

25. Declining insulin requirement in the late first trimester of diabetic pregnancy / Jovanovic L. [et al.] ; National Institute of Child Health and Human Developtment, Diabetes in Early Pregnancy Study Group // Diabetes Care. - 2001. - Vol. 24, iss. 7. - P. 1130-1136. - https:// doi.org/10.2337/diacare.24.7.1130.

26. Pharmacokinetics and pharmacodynamics of subcutaneous injection of long-acting insulin analog glargine, NPH insulin and ultralente human insulin and continuous subcutaneous infusion of insulin lispro / M. Lepore [et al.] // Diabetes. - 2000. - Vol. 49, iss. 12. - P. 2142-2148. https://doi.org/10.2337/diabetes.49.12.2142.

27. Preprandial versus postprandial blood glucose monitoring in type 1 diabetic pregnancy: a randomized controlled clinical trial / J. G. Manderson [et al.] // Am. J. Obstet. Gynecol. - 2003. - Vol. 189, iss. 2. - P. 507-512.

28. Glycosylated hemoglobin as predictor of adverse fetal outcome in type 1 diabetic pregnancies / G. L. Nielsen [et al] // Acta Diabet. - 1997. - Vol. 34, iss. 3. - P. 217-222.

29. Performance of the continuous glucosemonitoring system (CGMS) during development of ketosis in patients on insulin pump therapy / J. Pfutzner [et al.] // Diabet. Med. - 2006. - Vol. 23, iss. 10. - P. 1124-1129. - doi: 10.1111/j.1464-5491.2006.01945.x.

30. Use of insulin glargine during pregnancy / Pöyhönen-Alho M. [et al.] // Acta Obs. Gyn. Scand. - 2007. - Vol. 86, iss. 10. - P. 1171-1174. - doi: 10.1080/00016340701515282.

31. Screening for gestational diabetes mellitus: U.S. Preventive Services Task Force recommendation statement / Preventive Services Task Force // Ann. Intern. Med. - 2008. - Vol. 148, iss. 10. - P. 759-765.

32. Price, N. Use of insulin glargine during pregnancy: a casecontrol pilot study / N. Price, C. Bartlett, M. D. Gillmer // BJOG. - 2007. - Vol. 114, iss. 4. - P. 453-457. - doi: 10.1111/j.1471-0528.2006.01216.x.

33. Ramin, K. D. Diabetic ketoacidosis in pregnancy / K. D. Ramin // Ob-stet. Gynecol. Clin. Noth. Amer. - 1999. - Vol. 26, iss. 3. - P. 481-487. - https://doi. org/10.1016/S0889-8545(05)70092-9.

34. Continuous glucose monitoring for the evaluation of gravid women with type 1 diabetes mellitus / Y. Yogev [et al.] // Obstet. Gynecol. - 2003. - Vol. 101, iss. 4. P. 633-638.

\section{References}

1. Arbatskaya NYu. Sakharnyj diabet i beremennost. Farmateka. 2000;5:30-36. (Russian).

2. Arzhanova ON, Kosheleva NG. Osobennosti techeniya beremennosti i rodov pri sakharnom diabete $\mathrm{v}$ sovremennykh usloviyakh. Zhurnal akusherstva $i$ zhenskikh boleznej. 2006;1:12-16. (Russian).

3. Grigoryan OR, Sheremeteva EV, Andreeva EN, Dedov II. Planirovanie beremennosti u zhenshhin s sakharnym diabetom. Vestnik reproduktivnogo zdorovya. 2011;2:23-31. 
(Russian)

4. Danilova LI, Burko II, Zabarovskaya ZV. Sakharnyj diabet i beremennost. Minsk; 2010. 34 p. (Russian).

5. Dedov II, Shestakova MV. Sakharnyj diabet: retinopatiya, nefropatiya. Moskva: Meditsina; 2001. 176 p. (Russian).

6. Dedov II. Shestakova MV, Galstjan GR, Grigorjan OR, Esajan RM, Kalashnikov VJu, Kuraeva TL, Lipatov DV, Majorov AJu, Peterkova VA, Smirnova OM, Starostina EG, Surkova EV, Suhareva OJu, Tokmakova AJu, Shamhalova MSh, Jarek-Martynova IR. Algoritmy spetsializirovannoj meditsinskoj pomoshhi bolnym sakharnym diabetom. Sakharnyj diabet. 2015;18(1S):72-74. (Russian).

7. Demidova IYu, Arbatskaya NYu, Melnikova EP. Aktualnye problemy kompensatsii cakharnogo diabeta pri beremennosti. Sakharnyj diabet. 2009;4:32-38. (Russian).

8. Zakharova TG, Petrova MM, Laka GP. Sakharnyj diabet i beremennost: ucheb. posobie. Krasnoyarsk: Izdatelskie linii; 2006. 126 p. (Russian).

9. Potin VV, Borovik NV, Tiselko AV. Insulinoterapiya bolnykh sakharnym diabetom 1 tipa vo vremya beremennosti. Sakharnyj diabet. 2009;1:39-41. (Russian).

10. Fedorova MV, Krasnopolskij VI, Petrukhin VA. Sakharnyj diabet, beremennost i diabeticheskaya fetopatiya. Moskva: Meditsina; 2001. 288 p. (Russian).

11. Diabet i beremennost: obzor zapadnyh klinicheskih rekomendacij UpToDate.com. Chernov D, translator. 2015. 118 p. (Russian).

12. American Diabetes Association. Standards of medical care in diabetes-2014. DiabetesCare. 2014;37(1):S14-80. doi: $10.2337 / \mathrm{dc} 14-\mathrm{S} 014$.

13. DCCT Research Group. Effects of pregnancy on microvascular complications in the Diabetic Control and Complications Trial. Diabet Care. 2000;23:1084-1100.

14. Crowe SM, Mastrobattista JM, Monga M. Oral glucose tolerance test and the preparatory diet. Am. J. Obstet. Gynecol. 2000;182(5):1052-1054.

15. Di Cianni G, Torlone E, Lencione C, Bonomo M, Di Benedetto A, Napoli A, Vitacolonna E, Mannino D, Lapolla A. Perinatal outcomes associated with the use of glargine in pregnancy. Diabet Med. 2008;25(8):993-996. doi: 10.1111/j.1464-5491.2008.02485.x.

16. Evers IM, de Valk HW, Mol BWJ, ter Braak EWMT, Visser GHA. Macrosomia despite good glycaemic control in Type I diabetic pregnancy; results of a nationwide study in the Netherlands. Diabetologia. 2002;45(11):14841489. doi: 10.1007/s00125-002-0958-7.

17. The Task Force on the Management of Cardiovascular Diseases During Pregnancy of the European Society of Cardiology. Expert consensus document on management of cardiovascular diseases during pregnancy. Eur. Heart $J$. 2003;24(11):761-781.

18. Gabbe SG, Holing E, Temple P, Brown ZA. Benefits, risks, costs, and patients satisfaction associated with insulin pump therapy for the pregnancy complicated by type $1 \mathrm{di}-$ abetes mellitus. Am. J. Obstet Gynecol. 2000;182(6):1283.

19. Gallen IW, Jaap AJ, Roland JM, Chirayath IW. Survey of glargine use in 115 pregnant women with type 1 diabetes. Diabet Med. 2008:25(2):165-169.

20. Hellmuth E, Damm P, Molsted-Pedersen L, Bendtson I. Prevalence of nocturnal hypoglycemia in first trimester of pregnancy in patients with insulin treated diabetes mellitus. Acta Obs. Gyn. Scand. 2000;79(11):958. doi: 10.1034/j.1600-0412.2000.079011958.x.

21. Holing EV, Beyer CS, Brown ZA, Connell FA. Why dont women with diabetes plan their pregnancies? Dia- betes Care. 1998;21(6):889-895. https://doi.org/10.2337/ diacare.21.6.889.

22. Home P, Lindholm A, Hylleberg B, Round P. Improved glycemic control with insulin aspart: a multicenter randomized double-blind crossover trial in type 1 diabetic patients. UK Insulin Aspart Study Group. DiabetCare. 1998;21(11):1904-1909. https://doi.org/10.2337/ diacare.21.11.1904.

23. Inkster ME, Fahey TP, Donnan PT, Leese GP, Mires GJ, Murphy DJ. Poor glycatedhaemoglobin control and adverse pregnancy outcomes in type 1 and type 2 diabetes mellitus: Systematic review of observational studies. BMC Pregnancy and Childbirth. 2006.6:30. http://doi. org/10.1186/1471-2393-6-30.

24. Janz NK, Herman WH, Becker MP, Charron-Prochownik D, Shayna VL, Lesnick TG, Jacober SJ, Fachnie JD, Kruger DF, Sanfield JA, Rosenblatt SI, Lorenz RP. Diabetes and pregnancy: factors associated with seeking preconception care. Diabetes Care. 1995;18(2):157-165. https:// doi.org/10.2337/diacare.18.2.157.

25. Jovanovic L, Knopp RH, Brown Z, Conley MR, Park E, Mills JL, Metzger BE, Aarons JH, Holmes LB, Simpson JL; National Institute of Child Health and Human Developtment, Diabetes in Early Pregnancy Study Group. Declining insulin requirement in the late first trimester of diabetic pregnancy. Diabetes Care. 2001;24(7):1130-1136. https://doi.org/10.2337/diacare.24.7.1130.

26. Lepore M, Pampanelli S, Fanelli C, Porcellati F, Bartocci L, Di Vincenzo A, Cordoni C, Costa E, Brunetti P, Bolli GB. Pharmacokinetics and pharmacodynamics of subcutaneous injection of long-acting insulin analog glargine, NPH insulin and ultralente human insulin and continuous subcutaneous infusion of insulin lispro. Diabetes. 2000;49(12):2142-2148. https://doi.org/10.2337/ diabetes.49.12.2142.

27. Manderson JG, Patterson CC, Hadden DR, Traub AI, Ennis C, McCance DR. Preprandial versus postprandial blood glucose monitoring in type 1 diabetic pregnancy: a randomized controlled clinical trial. Am. J. Obstet. Gynecol. 2003;189(2):507-512.

28. Nielsen GL, Sorensen HT, Nielsen PH, Sabroe S, Olsen J. Glycosylated hemoglobin as predictor of adverse fetal outcome in type 1 diabetic pregnancies. Acta Diabet. 1997;34(3):217-222.

29. Pfutzner J, Forst T, Butzer R, Forst S, Weber MM, Pfützner AH, Pfützner A. Performance of the continuous glucosemonitoring system (CGMS) during development of ketosis in patients on insulin pump therapy. Diabet. Med. 2006;23(10):1124-1129. doi: 10.1111/j.1464-5491.2006.01945.x.

30. Pöyhönen-Alho M, Rönnemaa T, Saltevo J, Ekblad U, Kaaja RJ. Use of insulin glargine during pregnancy. Acta Obs. Gyn. Scand. 2007;86(10):1124-1129. doi: 10.1080/00016340701515282.

31. Preventive Services Task Force. Screening for gestational diabetes mellitus: U.S. Preventive Services Task Force recommendation statement. Ann. Intern. Med. 2008;148(10):759-765.

32. Price N, Bartlett C, Gillmer MD. Use of insulin glargine during pregnancy: a case-control pilot study. BJOG. 2007:114(4):453-457. https://doi: 10.1111/j.1471-0528.2006.01216.x.

33. Ramin KD. Diabetic ketoacidosis in pregnancy. Obstet. Gynecol. Clin. Noth. Amer. 1999;26(3):481-487. https:// doi.org/10.1016/S0889-8545(05)70092-9. 
34. Yogev Y, Chen R, Ben-Haroush A, Phillip M, Jovanovic

L, Hod M. Continuous glucose monitoring for the evaluation of gravid women with type 1 diabetes mellitus. Obstet. Gynecol. 2003;101(4):633-638.

\title{
DIABETES MELLITUS AND PREGNANCY. PART II. PECULIAR PROPERTIES, MANAGEMENT OF PATIENTS
}

\author{
'Nikonova L. V., 'Tishkovskiy S. V., ' Hadomskaya V. I., 'Davydchyk E. V., \\ ${ }^{1}$ Gulinskaya O. V. \\ 'Educational Institution «Grodno State Medical University», Grodno, Belarus \\ 2"City Polyclinic N4", Grodno, Belarus
}

Background: diabetes mellitus is one of the most widespread diseases in the world and one of those which lead to complications. One of the factors, which influence the clinical course of diabetes mellitus and its complications is pregnancy.

Aim: to study the peculiarities of diabetes mellitus clinical course and its complications in pregnancy as well as the management of diabetes mellitus in pregnant women depending on the gestational age, clinical course of diabetes mellitus and its complications.

Material and methods: 34 sources of literature by native and foreign authors on the studied subject have been analyzed.

Results: it has been established that the clinical course of diabetes mellitus in pregnancy is labile and contributes to the development of vascular complications in a mother. Strict control of glycaemia, the introduction of a regimen of multiple insulin injections have a positive effect on the clinical course of pregnancy and birth of a healthy child.

Conclusion: the application of intensified insulin therapy in pregnant women with diabetes mellitus, improved management and treatment of pregnant women, application of modern technologies have given opportunities to improve pregnancy outcomes in a mother and a child: the frequency of severe forms of gestosis, hydramnion, premature birth, perinatal mortality has decreased.

Keywords: diabetes mellitus, pregnancy, hyperglycemia, hypoglycemia, insulin therapy, treatment.

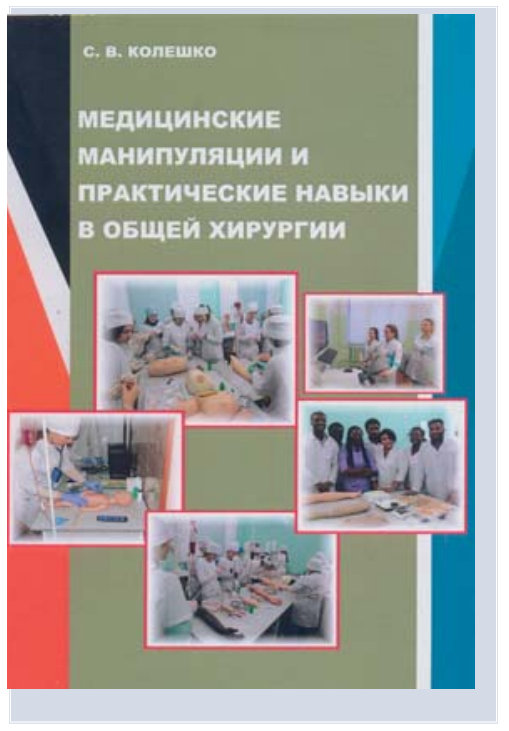

Медицинские манипуляции и практические навыки в общей хирургии : учебное пособие для студентов лечебного (специальность 1-79 0101 "Лечебное дело"), педиатрического (специальность 1-79 0102 "Педиатрия"), медико-психологического (специальность 1-79 0105 "Медико-психологическое дело") факультетов / С. В. Колешко ; Министерство здравоохранения Республики Беларусь, Учреждение образования "Гродненский государственный медицинский университет", Кафедра общей хирургии. - Гродно : ГрГМУ, 2017. - 106 с. : рис. - Библиогр.: c. 106. - ISBN 978-985-558-854-3.

Данное пособие составлено в соответствии с учебной программой и рекомендуется к использованию при освоении практических навыков, при подготовке к занятиям и курсовому экзамену. Описание каждого практического навыка включает подробный алгоритм с четкой последовательностью действий и их иллюстрацией. Все рисунки взяты из свободного доступа в интернете.

Предназначено для студентов лечебного, педиатрического, медико-психологического факультетов. 